\title{
System for psychophysical measurements of two-photon vision
}

\author{
A. Zielińska, ${ }^{1}$ K. Kiluk, ${ }^{2}$ M. Wojtkowski, ${ }^{1,2,3}$ and K.. Komar, ${ }^{* 1,2}$ \\ ${ }^{1}$ Institute of Physics, Faculty of Physics, Astronomy and Informatics, Nicolaus Copernicus University, \\ Grudziadzka 5, 87-100 Toruń, \\ ${ }^{2}$ Baltic Institute of Technology, Al. Zwycięstwa 96/98, 81-451 Gdynia, \\ ${ }^{3}$ Institute of Physical Chemistry, Polish Academy of Sciences, Kasprzaka 44/52, 01-224 Warsaw
}

Received August 20, 2018; accepted March 26, 2019; published March 31, 2019

\begin{abstract}
Two-photon absorption occurring in photoreceptors of the human eye is responsible for the perception of pulsed infrared laser beams. The color of perceived light corresponds to about half of the wavelength of a stimulating beam. This recently discovered phenomenon is called two-photon vision. However, it is yet not clear how such phenomena could impact an ophthalmic diagnosis and therefore further studies are required. In this paper we present the system that enables future psychophysical experiments to extend our knowledge about two-photon vision and its diagnostic value by identifying differences between two-photon and single-photon vision.
\end{abstract}

The range of electromagnetic radiation perceived by the human eye is commonly called "visible range" and determined to $380 \div 780 \mathrm{~nm}$ [1]. However, it has been observed recently that pulsed laser beams in a range of near-infrared wavelengths $(900 \div 1200 \mathrm{~nm})$ are also perceived by the human eye and that colors of these beams corresponded approximately to half of their wavelength. Detailed investigations of this phenomenon led to the conclusion that it is caused by two-photon absorption taking place in photoreceptors located in the inner layers of the retina [2]. A further study [3] compared the visual acuity mediated by two-photon vision with that of single-photon vision. The spatial properties proved to be the same for normal and for twophoton vision. Other interesting studies include the measurements of visual sensitivity thresholds [4] and twophoton response to dark-adaptation performed in selected retinal locations [5]. There are still many unknows in this area. The aim of future research is to understand twophoton vision and to determine how it differs from singlephoton absorption. The far-reaching goal of these studies is to verify the applicability of two-photon vision to ophthalmic diagnosis. In this report we present the system for psychophysical measurements of twophoton vision that can be applied for in vivo human studies. We also demonstrate exemplary results of darkadapted visual thresholds measured by our set-up.

The system for psychophysical measurements of twophoton vision is presented in Fig. 1. The set-up combines four different optical modules: stimulus delivery (laser beams of wavelengths $520 \mathrm{~nm} / 1040 \mathrm{~nm})$, pupil monitoring $(940 \mathrm{~nm})$, fixation $(630 \mathrm{~nm})$ and bleaching/background

*E-mail: kkomar@fizyka.umk.pl illumination (visible range). The spectral characteristics of light used for each module are shown in Fig. 2.

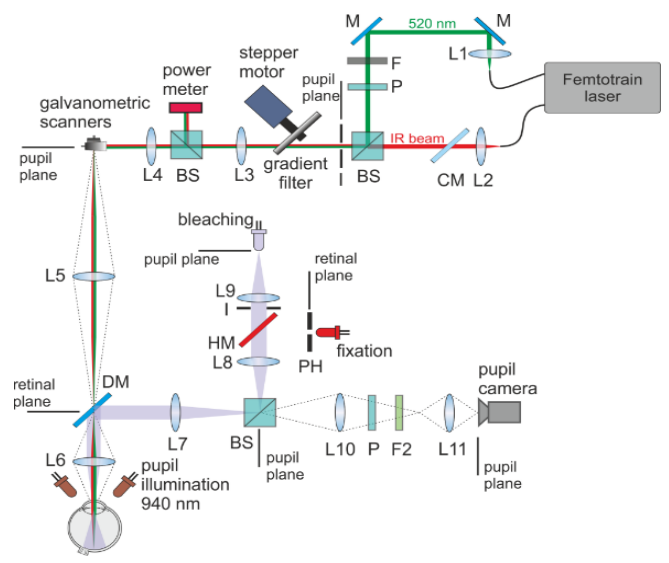

Fig. 1. Optical system dedicated to two-photon vision measurements. Legend: BS - beam splitter, M - mirror, DM - dichroic mirror, CM cold mirror, HM - hot mirror, P - polarizer, F- filter, L - lens, PH pinhole, I - iris.

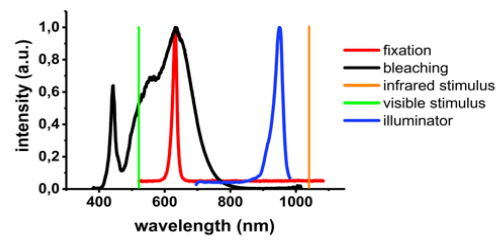

Fig. 2. The wavelengths used in two-photon vision measurements.

The stimulus delivery module uses Gaussian beams from a Femtotrain laser (HighQ Lasers) with a pulse length of $200 \mathrm{fs}$ and repetition rate of $76 \mathrm{MHz}$. Due to the dispersion in optical fibers transmitting light to the set-up, the pulse width at the subject's pupil was elongated to approx. $1 \div 2 \mathrm{ps}$. The optical power range at the pupil plane of the subject's eye was $200 \mathrm{nW} \div 1 \mathrm{~mW} \quad(17.6 \mathrm{log}$ photons $\cdot \mathrm{s}^{-1} \cdot \mathrm{cm}^{-2}$ ) for $1040 \mathrm{~nm}$ and several $\mathrm{fW} \div 8.5 \mathrm{nW}$ $\left(12.2 \log\right.$ photons $\left.\cdot \mathrm{s}^{-1} \cdot \mathrm{cm}^{-2}\right)$ for $520 \mathrm{~nm}$. The collimators for infrared and visible beams consist of SM optical fibers and lenses L1, L2 (both 19mm). In the IR path there is an additional cold mirror $\mathrm{CM}$ to cut off the visible light. A neutral density filter is used to attenuate the visible light by a factor of $1.2 \times 10^{-4}$ for $520 \mathrm{~nm}$. Both stimulus beams 
are combined into one coaxial path by a beam splitter BS (BS017, Thorlabs). The regulated iris I is mounted to adjust the diameters of both beams to the same size. Typically, after passing through, the beam diameters equal $3.7 \mathrm{~mm}\left(1 / \mathrm{e}^{2}\right)$. Next, the beam is directed into galvanometer XY scanners (Cambridge Technology) through the first telescope (L3 and L4, 50mm, both). After reflecting off the pair of galvo-scanners, the parallel beam is directed through the second telescope composed of L5 $(150 \mathrm{~mm})$ and L6 $(50 \mathrm{~mm})$, into the subject's pupil and is focused on the retina by the natural optical system of the subject's eye. The beam diameter on the cornea is equal to $1.3 \mathrm{~mm}$.

For visual threshold measurement, the subject can adjust the power of an incident beam by scrolling the computer mouse linked to a motorized NDF gradient filter which is placed before L3. The actual beam power is measured by a power meter PM placed between L3 and L4.

Galvo-scanners enable projecting the different shapes of optotype on the subject's retina (f.e. donut, cross, circle, point) at different retinal positions. It is also possible to set the time of displaying optotype and time when it is off (f.e. for $200 \mathrm{~ms}$ stimulus is on and afterwards for $600 \mathrm{~ms}$ stimulus is off). The setpoint for scanners equal to $1.46 \mathrm{~V}$ allows to obtain the maximum range of retina scanning due to a limited aperture of the optics path. The maximum angle $\left(\alpha_{\text {mech }}\right)$ is equal to $2.2^{\circ}$ and corresponds to half of the optical angle $\left(\alpha_{o p t}\right.$, between the stimulus beam reflected from the scanners and optical axis) which is $4.4^{\circ}$. The corresponding visual angle of the stimuli subtend at the pupil plane $\left(\alpha_{\text {eye }}\right)$ is $26.0^{\circ}$. The voltage ( $V$, expressed in $\mathrm{V}$ ) set to galvo-scanners depends on the angle of stimuli in the eye:

$$
V=19 \cdot \frac{f_{6}}{f_{5}} \cdot \tan \frac{\alpha_{\text {eye }}}{2},
$$

where $f_{5}, f_{6}$ are the focal lengths of the respective lenses expressed in $\mathrm{mm}, \frac{\alpha_{\text {eye }}}{2}$ is a half of the angle between the beam entering the eye at the pupil plane and the optical axis.

In the pupil monitoring module the eye is illuminated by 16 infrared diodes $(940 \mathrm{~nm})$ mounted radially on a ring placed on a tube holding the last lens of the system (L6). The maximal value measured at the pupil plane is equal to $1.55 \mathrm{~mW}$. The eye pupil plane is conjugated with a camera matrix by two telescopes L6, L7 and L10 (125mm), L11 (19mm). A polarizer P (LPNIRE100B, Thorlabs) is used to minimize the reflections of laser stimulus beams from the surface of the cornea, and a low pass filter (ET750LP, Chroma) to suppress the reflections from a bleaching light source. The pupil diameter and its position is monitored by an USB CMOS camera (DCC1545M, Thorlabs). The recorded image consists of $512 \times 512$ pixels.

The eye position during measurement is stabilized by a fixation point - red LED diode $(630 \mathrm{~nm})$ with a $150 \mu \mathrm{m}$ pinhole $\mathrm{PH}$ placed in the plane conjugated to the retina by telescope L8 $(100 \mathrm{~mm})$ and L7 (75mm) and, further, lens L6 and lens of the subject's eye. The pinhole is mounted on the XY stage to allow changing the location of a fixation point at the retinal plane for stimulation of different retinal areas.

The optical path of bleaching light delivery includes a white LED diode (MWWHL3, Thorlabs) which provides uniform illumination of the subject's retina in the Maxwellian view [6]. It allows to measure the recovery of light sensitivity after variable visible light bleaching. The optical path includes also two telescopes of L9 (30mm), L8 and L7, L6 and an eye lens which is the last lens in the Maxwellian view system. Additionally, the regulated aperture I inserted before bleaching LED allows to change the size of a circle. Telescopes create a source image (bleaching) at the pupil plane. According to [6] the area of illuminated retinal field $S_{R}$ in this complex Maxwellian view system could be calculated from Eq. 2:

$$
S_{R}=S_{\text {stop }} \cdot\left(\frac{f_{\text {eye }} f_{7}}{f_{6} f_{8}}\right)^{2},
$$

where $S_{\text {stop }}$ is the field stop in the aperture of L9 or in the regulated aperture I after L9, $f_{\text {eye }}$ is the focal length of the eye and $f_{6} f_{7}, f_{8}$ are the focal lengths of the respective lenses.

Taking $f_{\text {eye }}$ equal to $16.67 \mathrm{~mm}$ for the schematic eye and assuming the maximum area of a field stop as a clear aperture of lens L9 equal to $23 \mathrm{~mm}$ in diameter and thus area $415.5 \mathrm{~mm}^{2}$, the illuminated retinal field is about $27.0 \mathrm{~mm}^{2}$. This value corresponds to the diameter of bleaching at a retinal plane of $5.9 \mathrm{~mm}\left(19.6^{\circ}\right)$. For smaller aperture exemplary of the size corresponding to a circle optotype $(0.2 \mathrm{~V})$ which has a diameter equal to $4.1 \mathrm{~mm}$ we get a retinal illuminated field about $0.8 \mathrm{~mm}^{2}$. The diameter of bleaching at the retinal plane is $1.0 \mathrm{~mm}\left(3.33^{\circ}\right)$.

The retinal illuminance possible to obtain for a bleaching purpose in our system is in a range from 0 to $4.85 \cdot 10^{7} \mathrm{Td}-$ measured in accordance with the procedure from [7]. Bleaching LED light could be used also to create a uniform circle background of different colors by regulating the amount of light and by using the filters transmitting selected part of the spectrum, respectively.

During all tests the subject sits and maintains his head on the chinrest motorized in two dimensions (height adjustment is carried out manually). The correction of the subject's pupil position is carried out continuously by the person maintaining measurements. All powers of light incident on the eye were kept below the safety requirements of ANSI standards and the protocols were approved by the Internal Ethics Committee. Pupil dilation was not used.

The fixation and bleaching modules are connected by hot mirror HM (FM02R, Thorlabs) and introduced to the system by beam splitter BS (BS014, Thorlabs). These paths are coupled with a beam delivery path either 
by dichroic mirrors (DMSP650R, DMLP950R, Thorlabs) or a beam splitter (BSN10R, Thorlabs) selected depending on the stimulation beam used.

Dedicated LabVIEW programs support three different types of psychophysical experiments. The program for visibility thresholds measurement allows the subject to adjust the power of a stimulus. The program for dark adaptation measurement registers threshold values before the bleaching event of controlled duration appears. It also enables to register continuously the subject's thresholds until complete recovery. The program dedicated to monitoring pupil reaction records pupil images ( 20 per sec) and controls stimuli appearance. The pupil diameter and its position are determined in post-processing by a dedicated program written in MATLAB inspired by [8]. Figure 3a shows the consecutive steps of pupil image processing and Fig. $3 \mathrm{~b}$ shows the plot of the pupil diameter and pupil displacement registered during the experiments using $1040 \mathrm{~nm}$ stimulation with $600 \mu \mathrm{W}$. The stimulus displayed on the retina was a spiral donut of ext. radius $3.5^{\circ}$.
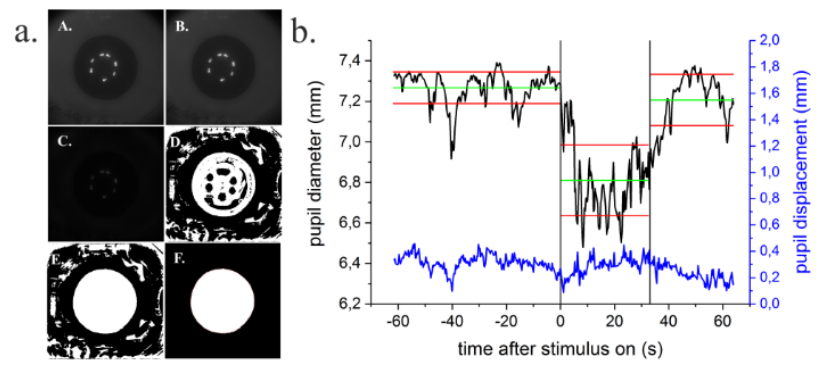

Fig. 3. Tools for the monitoring of pupil reaction: (a) Images illustrating subsequent algorithm steps: (A) original pupil image, (B) Gaussian filtration (smoothing), (C) equalization of histogram (contrast adjustment), (D) binarization, (E) filling by white holes, (F) deleting small objects and fitting circle to the pupil. (b) Plot showing the pupil diameter and pupil displacement. Green lines - mean of pupil diameter in specified time (before, during and after the stimulus), red lines - standard deviations. Mean values and SD are respectively $7.27 \pm 0.08 \mathrm{~mm}, 6.82 \pm 0.17 \mathrm{~mm}, 7.20 \pm 0.13 \mathrm{~mm}$.

After measurement, to estimate the size of an expected pupil size response to IR light, both stimuli were displayed and the power of 520nm stimulus was adjusted by the subject to obtain the same brightness as that of a $1040 \mathrm{~nm}$ beam. The adjusted brightness corresponds to the corneal flux of $\sim 9 \log$ photons $\cdot \mathrm{cm}^{2} \cdot \mathrm{s}$ for a fixed position of the beam. This weak stimulus may cause small changes in the pupil size [9]. Depending on the level of the subject's experience, registered reactions may be, to some extent, caused by both effects: pupil reaction to IR light and eye accommodation [10].

Exemplary results from dark-adapted visual thresholds [11] are shown in Fig. 4a. The sensitivity thresholds ratio between the fovea (more cones) and temporal retina (more rods) was 2.5-8 times smaller for $1040 \mathrm{~nm}$ beam than for a $520 \mathrm{~nm}$ one for 5 out of 6 subjects. It seems that cones are better stimulated by $1040 \mathrm{~nm}$ light. The comparison of the recovery curves after bleaching for visible and infrared beams is presented in Fig. 4b. The rod-cone interval for a two-photon stimulus was 0.8 order of magnitude smaller than for a single-photon and the rod-cone break occurred $\sim 2.5$ min later for an infrared beam. It suggests that a $1040 \mathrm{~nm}$ beam, although perceived as green, stimulates stronger cones comparing to rods than a $520 \mathrm{~nm}$ beam.
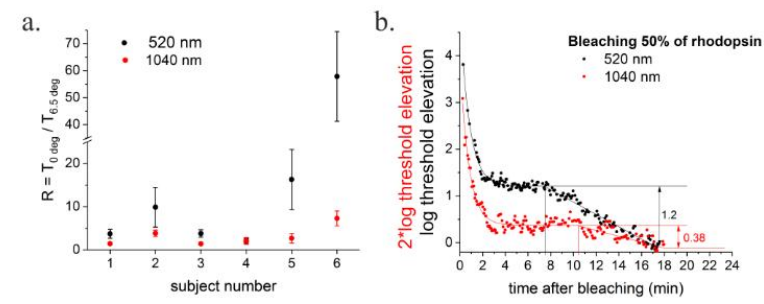

Fig. 4. Measurement of dark-adapted visual thresholds: (a) The ratio of darkadapted visual thresholds for six subjects for $520 \mathrm{~nm}$ and $1040 \mathrm{~nm}$ stimuli. Threshold were measured at the fovea and at $6.5^{\circ}$ temporal. Stimulus was a circle of size $0.5^{\circ}$ (b) Visual sensitivity threshold recovery after $150 \mathrm{~ms}$ bleaching $\left(47.6 \cdot 10^{6}\right.$ scotopic Td) for $1040 \mathrm{~nm}$ and $520 \mathrm{~nm}$ stimuli. Both reference values of threshold were calculated on the basis of 5 measurements before bleaching. Circle optotype of size $0.5^{\circ}$ was used to stimulate the temporal retina at $6.5^{\circ}$ from macula.

The presented results show the functionality of the system for psychophysical measurements of two-photon vision. The new area of knowledge dedicated to understanding two photon vision process will be explored using our system. Planned investigations allow to better characterize this vision and to evaluate its applicability in ophthalmic diagnosis.

This work was supported by the National Science Centre, project No. 2016/23/B/ST2/00752 and cofinanced by City of Gdynia, project No. 3/DOT/2016. M.W. would like to acknowledge the European Union Horizon 2020 research and innovation programme grant No. 666295 and the TEAM TECH/2016-3/20 programme of the Foundation for Polish Science co-financed by the European Union under the European Regional Development Fund.

\section{References}

[1] D.A. Atchison, G. Smith, Optics of the human eye (ButterworthHeinemann 2000).

[2] G. Palczewska et al., Proc. Nat. Academy of Sciences 111, 1428 (2014)

[3] P. Artal, S. Manzanera, K. Komar, A. Gambín-Regadera, M. Wojtkowski, Optica 4, 1488 (2017).

[4] K. Komar et al., Investigative Ophthalmology and Visual Science 57, 196 (2016).

[5] K. Komar et al., Investigative Ophthalmology and Visual Science 59, 4049 (2018).

[6] S.A. Burns, R.H. Webb, Handbook of optics (New York, McGraw Hill 1994).

[7] R.W. Nygaard, T. E. Frumkes, Vision Research (1982).

[8] P. Melillo et al., Biomedical engineering online 11, 40 (2012).

[9] P.A. Stanley, A.K. Davies, Ophthalmic and Physiological Optics, 15, 601 (1995)

[10] S. Mathôt, Journal of Cognition 1 (2018).

[11] M.M. Thomas, T.D. Lamb, J. Physiology 518, 479 (1999). 